HU-EP-05/35

hep-th/0508071

\title{
D-branes in overcritical electric fields
}

\author{
Harald Dorn, Mario Salizzoni and Alessandro Torrielli \\ Humboldt-Universität zu Berlin, Institut für Physik \\ Newtonstraße 15, D-12489 Berlin \\ dorn, sali, torriell@physik.hu-berlin.de
}

\begin{abstract}
We collect some arguments for treating a D-brane with overcritical electric field as a well-posed initial condition for a D-brane decay. Within the field theoretical toy model of Minahan and Zwiebach we give an estimate for the condensates of the related infinite tower of tachyonic excitations.
\end{abstract}




\section{Introduction}

Tachyon dynamics in the context of D-brane decay was the subject of intense studies in recent years [1. The main focus has been on the field theoretical or string field theoretical aspects of tachyons as excitations indicating some instability leading to the decay of certain field configurations. This field theoretical point of view is by far more adequate than discussions in terms of a particle language including its conceptual problems with superluminal velocities.

Open strings in the presence of an overcritical electric field mainly have been considered as an ill-defined setting (there are however interesting connections with S-branes, see for instance [2, 3]). Classically the endpoints of open strings have to move with superluminal velocity and at the quantum level there appears a singularity at the critical field strength in the string analog of the Schwinger pair creation [4, [5]. On the other hand, looking at String Field Theory in the presence of an overcritical electric field presents a picture conceptually not so different from that with undercritical or zero electric field. Instead of one tachyonic excitation, accompanied by a zero mass and an infinite tower of stable excitations, one is faced with a stable excitation corresponding to the former tachyon and the fact that the infinite tower has become tachyonic. At this level the difference between undercritical and overcritical electric field seems to be only technical.

In the present paper we want to sketch this point of view in some more detail. Section 2 is devoted to a discussion of several aspects supporting the conjecture that the decay of D-branes due to the presence of an overcritical electric field can be a well posed problem in string theory. Of course any attempt to implement this picture has to handle the serious technical problems caused by the infinite number of tachyonic excitations driving the D-brane decay. The level truncation, which turned out to be very effective in the undercritical case, is no longer applicable. Some comments on the treatment of the overcritical case within Boundary Conformal Theory are also added. In Section 3 we then restrict ourselves to a discussion within a toy model introduced by Minahan and Zwiebach in 17, 18, accordingly modified by the presence of the overcritical electric field. As a quantitative result we will get an estimate of the values of all the fields in the infinite tachyonic tower after condensation.

\section{General considerations}

\subsection{Spectrum}

Propagation of open bosonic strings in the background of a constant electromagnetic field has been analyzed in a series of papers [4, 5, 6, 7]. The spectrum of the masses of a neutral string in the presence of a purely electric background has been derived as a particular case in [4, 17, where a classical instability has been shown to arise above a critical value of the electric field. In this case, string modes develop negative squared masses, and a tachyonic contribution to the mass comes also from the motion of the string in transverse directions. This classical instability, which is indeed present both for neutral and for charged strings, has no analogue in particle mechanics ${ }^{1}$. Its appearance is advocated in [4] as a signal of the fact that, in a second quantized treatment

\footnotetext{
${ }^{1}$ For charged strings, another kind of instability due to pair-production dominates at the quantum level for weak fields 4 5. This is the analog of the Schwinger phenomenon for particle electrodynamics. The derived rate is however zero for neutral strings, preventing this kind of mechanism from screening the growth in absolute value of the electric field, until it reaches the critical value at which the classical instability appears. On the other side, raising slowly $E$ exposes the bosonic D-brane to the usual tachyon decay (which is absent in the similar case of open superstrings). We assume an overcritical electric field as a given initial condition.
} 
of the theory, the string field would evolve away from the chosen unstable configuration. The spectrum can be reproduced by the formula

$$
k_{\mu} G^{\mu \nu} k_{\nu}=-(n-1)
$$

where we set $\alpha^{\prime}=1$. The metric to be used is the so called "open string metric"

$$
G^{\mu \nu}=\left(\frac{1}{g+B} g \frac{1}{g-B}\right)^{\mu \nu},
$$

where $g, B$ are the sigma-model (closed string) constant backgrounds [8]. This, in turn, is the natural formula arising from the analysis of the conformal dimensions of the vertex operators of the worldsheet conformal field theory [8], and consistently from evaluation of the singularities of string scattering amplitudes (see [9] and references therein). For our purposes we will take the closed string metric to be $g=\eta=(-1,1, \ldots, 1)$.

In the case of a purely electric background, without loss of generality one can choose the only non-zero components of the antisymmetric tensor to be

$$
B_{01}=-B_{10}=E \text {. }
$$

The open string metric becomes $G_{\mu \nu}=\left(-\left(1-E^{2}\right),\left(1-E^{2}\right), 1, \ldots, 1\right)$. One can notice the appearance of a critical value for the electric field: when $E=1$, the open string metric (2) changes its signature in the $(0,1)$ block. Formula (10) can be written as

$$
-k_{0}^{2}+k_{1}^{2}+\left(1-E^{2}\right) k_{\perp}^{2}=-\left(1-E^{2}\right)(n-1),
$$

or, equivalently,

$$
k_{\mu} \eta^{\mu \nu} k_{\nu}=-\left(1-E^{2}\right)(n-1)+E^{2} k_{\perp}^{2},
$$

$k_{\perp}$ indicating the momentum component transverse to the electric field. If one sets $k_{\mu} \eta^{\mu \nu} k_{\nu}=$ $-M^{2}$, this formula coincides with what is obtained for example in Sec. 4 of [7]. Generically, the distance between equidistant levels of the operator $M^{2}$ is smaller by a factor $\left(1-E^{2}\right)$ [7]. In the overcritical case, then one has $M^{2}<0$ (up to the first level due to presence of the zero-point energy of the oscillators), and a tachyonic contribution coming from the motion of the string in the transverse direction ${ }^{2}$.

What we can read out of (4), is that what was a time-like momentum (positive squared mass) in the absence of the background, becomes in the presence of an overcritical electric field a space-like one (tachyon). The spectrum of the bosonic open string has therefore been reversed, and it contains an infinite tower of tachyonic modes ${ }^{3}$.

It is also important to notice that a similar situation, in the presence of an electric background, would occur in superstring theory 4, 9, whose spectrum does not originally contain a tachyon. The appearance of an infinite tower of tachyons has no analog in the absence (or in the presence of an undercritical) electric field. Therefore, in the following we will regard the ensuing classical evolution away from the chosen configuration (which we will suggest to interpret as a decay of the electrified D25-brane) as due to the overcritical electric field [4].

\footnotetext{
${ }^{2}$ We keep in mind that this last contribution to the squared mass is present even for undercritical fields, while only for overcritical fields it gives a tachyonic contribution to the energy, according to the dispersion relation $k_{0}^{2}=k_{1}^{2}+\left(1-E^{2}\right) k_{\perp}^{2}+\left(1-E^{2}\right)(n-1)$ derived from (4). The sign of the "bare" squared mass term depending on the level $n$ is always reversed in overcritical fields.

${ }^{3}$ We remark that a possible interpretation of this change of signature from the point of view of the full target space could be ascribed to an interchange of the role of space and time between the directions 0 and 1 . However, if one demands now $x_{1}$ to assume the role of time, thus recovering a standard positive mass spectrum for the open string tower, then the closed string sector of the theory, whose masses are determined via the closed string metric as $\kappa_{\mu} g^{\mu \nu} \kappa_{\nu}=-4(n-1)$, would have infinite tachyons.
} 


\subsection{D-brane Decay}

The above mentioned instability is accompanied by another effect. When interpreting bosonic open string theory as a description of the dynamics of a space-filling D25-brane, the tension of such a brane is naturally derived from the Dirac-Born-Infeld action [11. Such a tension, in the presence of a constant electric field background, is therefore proportional to the Born-Infeld factor $\sqrt{1-E^{2}}$. This factor becomes imaginary in the overcritical case, which is to say that the squared mass of the brane becomes negative.

We can interpret this occurrence in the open string picture. Classically, an electric field stretches open strings against their internal tension [10]. At the critical value the electric force counterbalances the tendency of the string to oscillate, and stretches it to infinity. Beyond the critical value the classical tachyonic instability is generated ${ }^{4}$. This is a signal that the nonperturbative string field theory vacuum, whose dynamics is determined by the open string theory defined on such a background, acquires a tachyonic instability. The fact that the D25brane becomes tachyonic has another manifestation in the superluminal velocity of the T-dual D24-brane [12. Equivalently, this is known to be a (tilted) S-brane, a spacelike object which arises in the description of the standard D-brane decay via the rolling tachyon picture [2, 3. In particular, in [2] an effective action has been derived for S-branes, which turns out to be equal to the Dirac-Born-Infeld action times a factor $i$.

We suggest to interpret the features of the first quantized spectrum discussed in the previous section in the light of this physical picture. While it is by now clear how one should interpret the presence of the usual bosonic open string tachyon, which is responsible for driving the D-brane decay towards the closed string vacuum [1, here the situation is complicated by the fact that the brane itself behaves like a tachyonic soliton background of String Field Theory. This is precisely because, while the nature of the bosonic string tachyon is related to string zero point quantum oscillations without classical analogue, the tachyonic tower described in the previous section reflects the classical instability of the tachyonic brane. What we propose is therefore that, still, the idea of describing the decay through the rolling of the tachyonic excitations living on the worldvolume is applicable, with the natural difference being represented by the presence of an infinite number of tachyonic states. The practical way to do this will be described in the next section.

\subsection{String Field Theory and BCFT}

The main tool to study the rolling of the tachyon has been Cubic String Field Theory [13], in connection with methods of Boundary Conformal Field Theory [1]. On one side, the presence of a minimum of the String Field Theory potential corresponding to the absence of perturbative open string excitations has been firmly established in the level truncation scheme. On the other side, an exactly marginal boundary perturbation to the world-sheet action for the tachyon profile of the form

$$
\lambda \int_{\partial \Sigma} \cosh \left[X_{0}(t)\right] d t
$$

has been used [1] to describe the time evolution of the system and to derive the related stressenergy tensor during the decay. In the presence of an undercritical electric field, the procedure has been generalized in [14. The final products of the decay include in this case the additional presence of fundamental string charges in the tachyon vacuum.

\footnotetext{
${ }^{4}$ At the same time the endpoints of the strings classically acquire a superluminal velocity [7.
} 
The idea is therefore to make use of the available formulation of String Field Theory in the presence of antisymmetric backgrounds, this time evaluated for an overcritical electric field. We still expect it to be the instrument which describes the nonperturbative decay of the original unstable configuration, through the evolution of the infinite tower of tachyons. However, if one tries to apply the above mentioned strategy to this new situation, one soon realizes the scarce suitability of the level truncation scheme ${ }^{5}$. Its good approximation relies on the fact that all the tower of positive mass excitations is stable around the origin (perturbative vacuum), and even if the higher spin fields assume non-zero vacuum expectation values at the closed string minimum [16], it is conceivable that these values will not differ too much from the original stable point at zero (this appears also as a feature of the toy model used in [17, 18, on which we will elaborate in the next section). Now instead, all of them are tachyonic at the origin, and one will expect all of them to substantially move away. Neglecting them all but a finite set, or in other words setting them to zero starting from a certain level on, does not look a priori like a good approximation.

On the other hand, since all the tower of states is rolling down, one would need like an infinite sum of boundary perturbations of the type (6) for the higher spin fields, with the suitable modifications in order to account for the electric field.

The boundary perturbation (6) was originally introduced by inspection of the linearized String Field Theory equations of motion. If one would like to proceed in analogous way, one has to look at solutions of the linearized equations of motion for the higher spin fields of the tower, in the background of an overcritical electric field. The main problem is the inclusion of all the tower, and we will sketch here only the proposal for the first state. For this purpose, we can follow the treatment reported in 19 to determine the condition for conformal invariance of the boundary coupling to the spin two field at the linearized level in the background field. Adopting their parameterization and choosing the gauge where the Stueckelberg field is set to zero, one obtains the term

$$
\int_{\partial \Sigma} A_{\mu \nu}(X) \partial^{a} X^{\mu} \partial_{a} X^{\nu} d t
$$

with the following conditions on the symmetric tensor $A_{\mu \nu}(X)$ :

$$
\left(\partial^{\sigma} \partial_{\sigma}-1\right) A_{\mu \nu}=0 ; \partial^{\mu} A_{\mu \nu}=0 ; A_{\mu}^{\mu}=0,
$$

where all indices are contracted with the open string metric. Taking this into account, and making a spatially independent ansatz, one finds that

$$
A_{\mu \nu}\left(X^{0}\right)=a_{\mu \nu} \cosh \left(X^{0} \sqrt{E^{2}-1}\right)
$$

is a solution, provided $a_{\mu \nu}$ is symmetric, purely spatial and traceless. Inserted in (17), the solution (9) provides a generalization of the boundary deformation (6) suitable for the new case. The polarization tensor $a_{\mu \nu}$ would have the same status as the parameter $\lambda$, the initial value of the tachyon.

This will have to be supplemented with all the remaining boundary couplings for the higher spin states. However, it can already be studied as a prototype in order to gain insight about the features of the boundary state resulting from such kind of perturbations, which will in turn determine the type of vacuum obtained at the end of the decay.

\footnotetext{
${ }^{5}$ See [15] for considerations on critical electric fields in Vacuum SFT.
} 

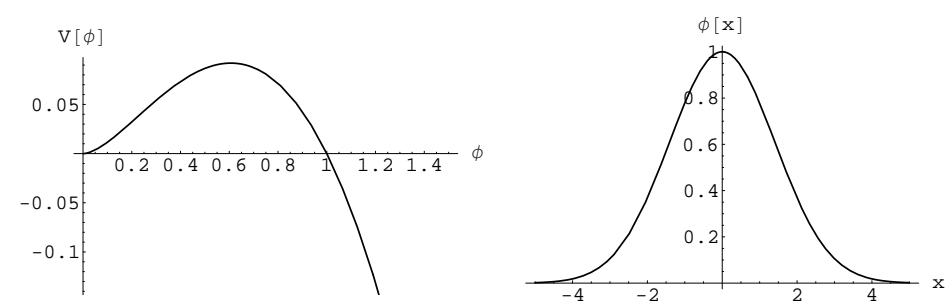

Figure 1: The shape of the potential $V(\phi)$ and of the lump solution.

\section{A Toy Model}

In [17, 18 toy models for the standard bosonic string tachyon condensation were studied, whose features simulate the behaviour found in String Field Theory for the true rolling tachyon. The idea is to study the lump solution of an effective field theory for the tachyon field. Fluctuations around this nonperturbative solution are determined by solving a Schrödinger equation of a known type, whose spectrum can be exactly computed resulting in an infinite tower of scalar excitations of increasing mass.

This situation has a close resemblance with the case of the String Field Theory obtained by quantization around the vacuum provided by a D-brane. In particular, the presence of a tachyon signals the instability of such a configuration, and the decay towards a stable minimum can be studied by looking for the minima of the obtained multiscalar potential. The main advantage is that in this simplified case one already knows the minimum of the original potential, and one can fix the values of the infinite set of scalar fields in the static case by simply requiring that summing the fluctuations to the lump profile produces the minimum of the original tachyon field. One can therefore treat simultaneously the whole tower of states, which in the case of an overcritical electric field is a strict requirement.

The technical procedure, in the case of absence of electric field, is briefly summarized in what follows. One considers the action for the tachyon field $\phi$ of a D25-brane

$$
S=\frac{1}{g_{o}^{2}} \int d^{25} y d x\left[-\frac{1}{2} \partial_{\mu} \phi \partial^{\mu} \phi-\frac{1}{2}\left(\partial_{x} \phi\right)^{2}-V(\phi)\right],
$$

where $y^{\mu}=(t, \vec{y})$ and $g_{o}$ is the open string coupling constant. The potential has the form shown in Fig.1:

$$
V(\phi)=-\frac{1}{4} \phi^{2} \ln \phi^{2}
$$

It has a maximum at $e^{-1 / 2}$, corresponding to the D25-brane, and a local minimum at 0 , corresponding to the closed string vacuum, where the curvature diverges. The last fact beautifully mimics the idea of decoupling of the open string degrees of freedom in the stable vacuum, which is a feature of the standard tachyon condensation. The potential admits a Gaussian lump solution independent of the $y$ variables

$$
\bar{\phi}(x)=\exp \left[-\frac{x^{2}}{4}\right],
$$

shown in Fig.1, that represents a D24-brane. After $\phi \rightarrow \bar{\phi}+\phi$, making the ansatz

$$
\phi=\sum_{n} \bar{\phi}_{n}(y) \psi_{n}(x)
$$


the computation of the spectrum of fluctuations around the lump is effectively reduced to the one dimensional Schrödinger equation for the harmonic oscillator

$$
-\frac{d^{2} \psi}{d x^{2}}+\left[-\frac{3}{2}+\frac{1}{4} x^{2}\right] \psi(x)=m^{2} \psi(x)
$$

that determines in this way the "open string" spectrum $m^{2}=n-1$ for $n \geq 0$.

The advantage of the model is that we know the minimum to be at the value where the original tachyon field $\phi$ is equal to 0 , therefore the exact values of the condensates for the whole tower are obtained by solving the following equation:

$$
0=\bar{\phi}(x)+\sum_{n} \bar{\phi}_{n} \psi_{n}(x)
$$

Since the Gaussian itself is the first eigenfunction of the harmonic oscillator, the solution of the equation is immediately found. It simply amounts to fixing the expectation value -1 for the tachyon $(n=0)$, and zero for all the other excitations. This means that only the tachyon condenses.

We want to use this model to describe the decay of a D-brane due to the overcritical electric field. The decay of the D-brane is now driven by the infinite tower of former massive fields now being tachyonic. We will see that it is still possible to study the decay of a D24-brane using the potential for the tachyon of a D25-brane.

Modifying the model in the presence of an electric field, simply amounts to use for the $y$ coordinates the open string metric, and simultaneously to introduce for them the star-product ${ }^{6}$.

Then, instead of (10), the action becomes

$$
S=\frac{1}{g_{o}^{2}} \sqrt{1-E^{2}} \int d^{25} y d x\left[-\frac{1}{2} G^{\mu \nu} \partial_{\mu} \phi \partial_{\nu} \phi-\frac{1}{2}\left(\partial_{x} \phi\right)^{2}-V(\phi)\right],
$$

where we included the factor $\sqrt{1-E^{2}}$ in order to recover the correct D-brane tension. Technically it would be equivalent to take into account the factor $\sqrt{-\operatorname{det} G}=\left|1-E^{2}\right|$ in the measure and to replace $g_{o}^{2}$ by $g_{o}^{2} \sqrt{-\operatorname{det}(g+B)}=g_{o}^{2} \sqrt{1-E^{2}}$, as is done for the DBI action in ref. [8]. Note that we insist on keeping the string coupling real for use in full String Field Theory. The action (16) is thought to be an effective one for the lowest string excitation. Having this in mind, the presence of an imaginary factor in (16) is no obstacle and in agreement with the DBI analysis.

The "transverse" part in the $x$ variable is not touched by the electric field. In particular, the same lump (12) is still a solution of the new equations of motion. With $G_{\mu \nu}$ from Sec.2, the action (16) can be written as

$$
\begin{aligned}
& S=\frac{1}{g_{o}^{2}} \frac{1}{\sqrt{1-E^{2}}} \int d^{25} y d x\left[\frac{1}{2}\left(\partial_{0} \phi\right)^{2}-\frac{1}{2}\left(\partial_{1} \phi\right)^{2}\right. \\
& \left.-\frac{1}{2}\left(1-E^{2}\right) \sum_{a}\left(\partial_{a} \phi\right)^{2}-\frac{1}{2}\left(1-E^{2}\right)\left(\partial_{x} \phi\right)^{2}-\left(1-E^{2}\right) V(\phi)\right] .
\end{aligned}
$$

Here, $a$ labels directions parallel to the brane, but perpendicular to the electric field.

Referring ourselves to the action (17), we first collected an overall factor in front, in order to make the following analysis clearer. We are interested in determining the solutions of the

\footnotetext{
${ }^{6}$ We will again always consider here either effective one dimensional $x$-variable problems, or static or linearized ones in the $y$ variables, therefore the star product will never play any role, and we will omit to write it.
} 


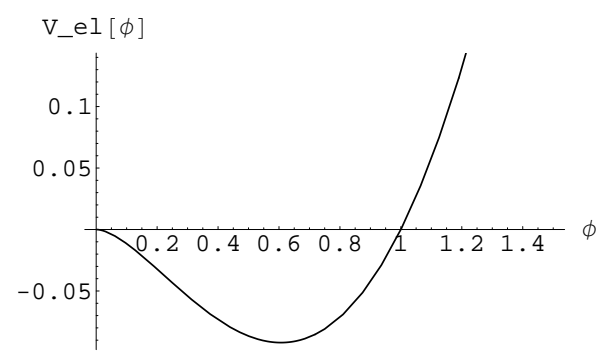

Figure 2: The shape of the potential $V_{e l}$ up to a factor $\left|1-E^{2}\right|$.

classical equations of motion, and to discuss their stability. The overall factor does not change this kind of analysis, since it does not alter the equations of motion and it does not affect the issue of stability around the extrema of the classical potential. We can therefore disregard this factor as far as this analysis is concerned. We will return later on its actual role.

From inspection of (17), one can see that the time derivative is normalized as in the $E=0$ case, but the potential has a factor in front with respect to that case, which changes sign in the overcritical case. We realize therefore that, together with the already known effect of the extra contribution coming from transverse motion ${ }^{7}$, the net effect of the overcritical electric field is to reverse the sign of the potential. This potential is drawn in Fig.2. Such an effect is consistent with the general argument concerning the sign of the squared masses in the overcritical electric background. The reversed tachyon potential for static configurations has a stable minimum at $e^{-1 / 2}$, that corresponds to the D25-brane. This means that the tachyon is now a stable (positive mass) state in the spectrum of the D25-brane ${ }^{8}$.

The model is therefore suitable to mimic the situation in the full String Field Theory, and one can be further convinced of this by examining the expanded action around the lump solution by replacing $\phi$ with $\bar{\phi}+\phi$. The action reads now

$$
\begin{aligned}
S= & \frac{1}{g_{o}^{2}} \sqrt{1-E^{2}} \int d^{25} y d x\left[-\frac{1}{2}\left(\frac{d \bar{\phi}}{d x}\right)^{2}-V(\bar{\phi})\right] \\
& +\frac{1}{g_{o}^{2}} \frac{1}{\sqrt{1-E^{2}}} \int d^{25} y d x\left[\frac{1}{2}\left(\partial_{0} \phi\right)^{2}-\frac{1}{2}\left(\partial_{1} \phi\right)^{2}-\frac{1}{2}\left(1-E^{2}\right) \sum_{a}\left(\partial_{a} \phi\right)^{2}\right. \\
& \left.-\frac{1}{2}\left(1-E^{2}\right) \phi\left(-\partial_{x}^{2}+V^{\prime \prime}(\bar{\phi})\right) \phi+\ldots\right] .
\end{aligned}
$$

The lump solution is still interpreted as a codimension 1 brane. Its asymptotes at $x= \pm \infty$ correspond to the absence of the D25-brane. The first two terms correctly reproduce the D24brane tension with the Born-Infeld factor which accounts for the electric background. This factor becoming imaginary was the object of the discussion in Sec.2.

The remaining part gives just the effective action for the fluctuations, which is used to determine the dynamics of the decay through the equations of motion and the analysis of stability, which are not influenced by the overall factor. The effective one dimensional Schrödinger problem is untouched by the presence of the electric field, the spectrum of fluctuations is therefore the same. But when we plug back the mode expansion in the action, we see that the eigenvalues

\footnotetext{
${ }^{7}$ Compare the derivatives in the direction $y^{a}$ in (17) with the contribution from $k_{\perp}$ in (4) and (5).

${ }^{8}$ The D25-brane is affected as well as the D24-brane (lump) by the electric field, which is switched on in directions common to both of them.
} 
of the Schrödinger equation contribute with a multiplicative factor $\left(1-E^{2}\right)$. This is precisely the modification of the squared mass for the open string spectrum according to the general treatment in Sec.2. We see here that the theory living on the worldvolume of the lump suitably describes the infinite tachyonic tower of states of an electrified D-brane. After integration over $x$, the resulting multiscalar potential can be taken as a toy model for the behaviour of the full String Field Theory in the presence of an overcritical electric field.

We can now predict inside this toy model the value of the condensate for the infinite tachyonic tower. The final stage representing the end of the rolling manifests itself in this toy model as a stable minimum of the reversed potential (corresponding to the old maximum) ${ }^{9}$.

We can apply the same technique as in the case without electric field to compute the expectation values, only requiring that the lump profile plus the fluctuations reduces the original tachyon to its true minimum at $e^{-1 / 2}$.

The overcritical counterpart of the equation (15) is therefore

$$
\begin{aligned}
e^{-1 / 2} & =\bar{\phi}(x)+\sum_{n=0}^{\infty} \bar{\phi}_{n} \psi_{n}(x) \\
& =\exp \left[-x^{2} / 4\right]+\sum_{n=0}^{\infty} \bar{\phi}_{n} \psi_{n}(x),
\end{aligned}
$$

where the eigenfunctions $\psi_{n}(x)$ are the usual harmonic oscillator basis constructed in terms of Hermite polynomials

$$
\psi_{n}(x)=\frac{1}{2^{n / 2} \sqrt{n !}} H_{n}\left(\frac{x}{\sqrt{2}}\right) \exp \left[-x^{2} / 4\right] .
$$

If we absorb the Gaussian profile in a redefinition of the coefficient $\bar{\phi}_{0}$, then Eq. (19) represents an expansion of a constant in terms of a Hilbert space basis. A constant is certainly not in the Hilbert space. Still it is possible to compute the coefficients $\bar{\phi}_{n}$ by performing the scalar product with any elements of the basis ${ }^{10}$. From

$$
\bar{\phi}_{n}=\frac{1}{\sqrt{2 \pi}} \int_{-\infty}^{\infty} d x \psi_{n}(x)\left(e^{-1 / 2}-\exp \left[-\frac{x^{2}}{4}\right]\right)
$$

one gets

$$
\bar{\phi}_{2 n+1}=0, \quad \bar{\phi}_{2 n}=-\delta_{n, 0}+\frac{2^{-n}}{n !} \sqrt{\frac{2(2 n) !}{e}} .
$$

This is the value of the condensates for the whole tower of states at the true vacuum. At large level number $n$, using Stirling's approximation, one gets a behaviour

$$
\bar{\phi}_{2 n} \longrightarrow \sqrt{\frac{2}{e}}(\pi n)^{-\frac{1}{4}}
$$

Due to the slow decrease of $\bar{\phi}_{2 n}$ in (23), the resulting series is not absolutely and not uniformly (for all $x$ ) convergent. However, the oscillations with $n$ of the sign of the Hermite polynomials enforce uniform convergence in finite $x$-intervals. It is straightforward to check this at least numerically.

\footnotetext{
${ }^{9}$ Of course it is open whether this feature is shared by the real String Field Theory potential. At this stage we take it as a conjecture.

${ }^{10}$ The basis is normalized such that $\int d x \psi_{n}(x) \psi_{m}(x)=\sqrt{2 \pi} \delta_{n, m}$.
} 
Although the expectation values for higher modes go to zero with $n \rightarrow \infty$ the decrease is not as fast as in the case without electric field [17, 18. In accordance with our previous discussion we take the slow decrease as an indication that level truncation would be a bad approximation. Indeed, we performed some explicit checks in level truncation, and they confirmed these expectations. Taking into account up to level $n=2$, and expanding the reversed effective potential up to the fifth power in these scalars, a minimum is found at $\bar{\phi}_{0}=-0.226, \bar{\phi}_{1}=0, \bar{\phi}_{2}=0.555$. This has to be compared with the exact result (22), namely $\bar{\phi}_{0}=-0.142, \bar{\phi}_{1}=0, \bar{\phi}_{2}=0.607$. At first sight this may seem not so bad an approximation, but we notice that, first, truncating at even powers up to the fourth, sixth and eighth ones produce instead no minimum, and, second, truncation to seventh and ninth powers produce a worse minimum. For example, with the seventh powers included the minimum is at $\bar{\phi}_{0}=-0.0831, \bar{\phi}_{1}=10^{-8}, \bar{\phi}_{2}=0.350$, and with the ninth powers included the minimum is at $\bar{\phi}_{0}=-0.0463, \bar{\phi}_{1}=10^{-4}, \bar{\phi}_{2}=0.259$. If convergence of level truncation is eventually to be obtained, it is done with wide oscillating behaviour, similar to the convergence of the sum in (19).

A last comment concerns the nature of the endpoint of the condensation process. The toy model setup is designed to simulate the decay of a D24-brane as a lump solution of the D25 tachyon field $\phi$. For the case of undercritical electric field the minimum of the potential for $\phi$ corresponds to the absence of the D25. Now in the overcritical case $\phi$ takes a value as in the presence of a D25. Within the lump-based model one has no possibility to decide whether this value for $\phi$ indicates a D25 or the true vacuum in which $\phi$ could assume just the same value $(\phi$ does not drive a D25 decay). However, beyond the model one should expect that it will be the duty of the tower of unstable D25 modes to prevent a formation of a D25.

From the open string point of view, the analogy with pair production of pointlike charges suggests that the endpoint of the condensation process corresponds to the discharge of the overcritical electric field: dipoles nucleating from the vacuum easily stretch to infinity until they screen the electric field making it critical or undercritical [3]. When looking at the D-brane decay, this means that the fluctuations around the unstable configuration are going to lower the value of the electric background itself towards critical or undercritical values. In the T-dual picture, one expects therefore that the T-dual superluminal brane (S-brane) would lower its velocity to (under-) luminal, thereby becoming a usual timelike D-brane. This would correspond to what is observed for example in 2, where the final stage of the S-brane evolution is represented by a flattening of its profile. This gives a hint that a remnant could be left after condensation, in accord to the general expectation of fundamental string fluxes as final products of the decay in the presence of electric fields [14. In order to get a full description of this phenomenon one would have to allow dynamics for the electric field as well and properly consider backreaction of the D-brane. In this respect we think that a closer connection to the S-brane picture could again be fruitful.

\section{Acknowledgments}

We thank Antonio Bassetto, Loriano Bonora, Giancarlo De Pol, Matthias Gaberdiel, Carlo Maccaferri, Adriano Parodi, Rodolfo Russo, Roberto Valandro and Hyun Seok Yang for useful discussions. We would like to thank Koji Hashimoto for pointing out to us the connection with S-branes, and for very interesting e-mail exchange. DFG supported H.D. and A.T. within the "Schwerpunktprogramm Stringtheorie 1096" and M.S. under the project SA 1356/1. 


\section{References}

[1] A. Sen, "Rolling tachyon," JHEP 0204 (2002) 048 arXiv:hep-th/0203211.

A. Sen, "Tachyon matter," JHEP 0207 (2002) 065 arXiv:hep-th/0203265.

A. Sen, "Tachyon dynamics in open string theory," arXiv:hep-th/0410103.

[2] K. Hashimoto, P. M. Ho and J. E. Wang, "S-brane actions," Phys. Rev. Lett. 90 (2003) 141601 arXiv:hep-th/0211090.

[3] K. Hashimoto, P. M. Ho, S. Nagaoka and J. E. Wang, "Time evolution via S-branes," Phys. Rev. D 68 (2003) 026007 arXiv:hep-th/0303172.

B. Durin and B. Pioline, "Aspects of Dirichlet S-branes," arXiv:hep-th/0507059.

[4] C. P. Burgess, "Open String Instability In Background Electric Fields," Nucl. Phys. B 294 (1987) 427.

[5] C. Bachas and M. Porrati, "Pair creation of open strings in an electric field," Phys. Lett. B 296 (1992) 77 arXiv:hep-th/9209032.

[6] E. S. Fradkin and A. A. Tseytlin, "Nonlinear Electrodynamics From Quantized Strings," Phys. Lett. B 163 (1985) 123.

A. Abouelsaood, C. G. . Callan, C. R. Nappi and S. A. Yost, "Open Strings In Background Gauge Fields," Nucl. Phys. B 280 (1987) 599.

[7] V. V. Nesterenko, "The Dynamics Of Open Strings In A Background Electromagnetic Field," Int. J. Mod. Phys. A 4 (1989) 2627.

[8] N. Seiberg and E. Witten, "String theory and noncommutative geometry," JHEP 9909 (1999) 032 arXiv:hep-th/9908142.

[9] A. Bassetto, A. Torrielli and R. Valandro, "One-loop unitarity of string theories in a constant external background and their Seiberg-Witten limit," JHEP 0401 (2004) 040 arXiv:hep-th/0311120.

[10] N. Seiberg, L. Susskind and N. Toumbas, "Strings in background electric field, space/time noncommutativity and a new noncritical string theory," JHEP 0006 (2000) 021 arXiv:hep-th/0005040.

[11] R. G. Leigh, "Dirac-Born-Infeld Action From Dirichlet Sigma Model," Mod. Phys. Lett. A 4 (1989) 2767.

J. Polchinski, "Lectures on D-branes," arXiv:hep-th/9611050.

[12] C. Bachas, "D-brane dynamics," Phys. Lett. B 374 (1996) 37 arXiv:hep-th/9511043.

[13] E. Witten, "Noncommutative Geometry And String Field Theory," Nucl. Phys. B 268 (1986) 253.

[14] P. Mukhopadhyay and A. Sen, "Decay of unstable D-branes with electric field," JHEP 0211 (2002) 047 arXiv:hep-th/0208142. 
[15] C. Maccaferri, R. J. Scherer Santos and D. D. Tolla, "Time-localized projectors in string field theory with E-field," Phys. Rev. D 71 (2005) 066007 arXiv:hep-th/0501011.

[16] V. A. Kostelecky and S. Samuel, "On A Nonperturbative Vacuum For The Open Bosonic String," Nucl. Phys. B 336 (1990) 263.

A. Sen and B. Zwiebach, "Tachyon condensation in string field theory," JHEP 0003 (2000) 002 arXiv:hep-th/9912249.

[17] B. Zwiebach, "A solvable toy model for tachyon condensation in string field theory," JHEP 0009 (2000) 028 arXiv:hep-th/0008227.

[18] J. A. Minahan and B. Zwiebach, "Field theory models for tachyon and gauge field string dynamics," JHEP 0009 (2000) 029 arXiv:hep-th/0008231.

[19] J. M. F. Labastida and M. A. H. Vozmediano, "Bosonic Strings In Background Massive Fields," Nucl. Phys. B 312 (1989) 308. 